\title{
LETTER TO THE EDITOR Persistent Elevation of Fetal Hemoglobin Following Chemotherapy in Sickle Cell Disease
}

To the Editor: Regulation of fetal hemoglobin ( $\mathrm{HbF}$ ) production is complex [1]. Our experience of persistent $\mathrm{HbF}$ elevation during a 7-year follow up in a child with homozygous sickle cell disease (SCD) who underwent chemotherapy for Wilms tumor (WT) adds to the complexity of the regulatory mechanisms in $\mathrm{HbF}$ production.

An 8-year-old African-American female homozygous SCD patient with metastatic WT was treated with vincristine, doxorubicin, and actinomycin D. After 6 months of therapy, she underwent nephrectomy; histology showed focal anaplasia. Interim, she developed a metastatic right liver lobe nodular lesion. She then received radiation to abdomen and lungs followed by ifosfamide, carboplatin, and etoposide chemotherapy. The treatment was completed in 26 months and her creatinine level remained normal. She did not require hospitalization for SCD complications and has had baseline hemoglobin of 9-10 g/dl. At the age of 3, her $\mathrm{HbF}$ was $17.6 \%$. Her parents do not have elevated $\mathrm{HbF}$ levels ruling out hereditary persistence of $\mathrm{HbF}$. Six months following treatment, her $\mathrm{HbF}$ was $33 \%$ along with increased red blood cell mean corpuscular volume (MCV) and mean corpuscular hemoglobin $(\mathrm{MCH})$. Despite $\mathrm{MCV}$ and $\mathrm{MCH}$ remaining stable, $\mathrm{HbF}$ trended down to $20.8 \%$ (Fig. 1). This may be secondary to agerelated increase in average $\mathrm{MCV}$ and $\mathrm{MCH}$ levels in children irrespective of the HbF. F cells shown by flow cytometry were 95 and $82 \%$ at 6 months and 6 years after the completion of chemotherapy, respectively.

Following chemotherapy, persistently high MCV has been reported in long-term follow up; however, $\mathrm{HbF}$ values have not been reported [2]. The relation between $\mathrm{HbF}$ and $\mathrm{F}$ cells in our case was within expected age-related distribution in SCD patients [3]. Elevation of $\mathrm{HbF}$ and $\mathrm{MCV}$ in this case is reminiscent of stress erythropoiesis. However, increased $\mathrm{HbF}$ persistence has been far long-lived in the absence of chemotherapy in contrast to the dependence of $\mathrm{HbF}$ elevation on the continuous administration of hydroxyurea in SCD. In addition to alterations in chromatin structure or kinetics of erythroid differentiation, alternative mechanisms have been identified in $\mathrm{HbF}$ induction [4,5]. Increased $\mathrm{HbF}$ is seen in juvenile myelomonocytic leukemia, which is associated with acquired mutations in the Ras/MAPK pathway. Stem cell factor induces adult erythroid growth and $\mathrm{HbF}$ production through Ras/MAPK pathway [6]. Elevated EPO is unlikely, since increases of EPO levels are transient following nephrectomy in kidney donors [7]. Hemoglobin levels were stable in our case.

The current case is unique in the prolonged elevation of $\mathrm{HbF}$. Persistent chemotherapy-related bone marrow microenvironment and cytokine milieu alterations may be an explanation. Emergence of an altered stem cell population secondary to acquired genetic or epigenetic changes resulting in a stress erythropoiesislike reaction might also be possible. In conclusion, prospective evaluation of similar cases may add to our knowledge of the regulation of $\mathrm{HbF}$ production.

Süreyya Savaşan, MD*

Carman and Ann Adams Department of Pediatrics Division of Hematology/Oncology Pediatric Blood and Marrow Transplant Program Children's Hospital of Michigan

Barbara Ann Karmanos Cancer Center Wayne State University School of Medicine Detroit, Michigan

Sharada A. Sarnaik, MD

Carman and Ann Adams Department of Pediatrics Division of Hematology/Oncology Sickle Cell Disease Center Children's Hospital of Michigan Barbara Ann Karmanos Cancer Center Wayne State University School of Medicine Detroit, Michigan
*Correspondence to: Süreyya Savaşan, MD, 3901 Beaubien Blvd., Children's Hospital of Michigan, Pediatric Blood and Marrow Transplant Program, Detroit, MI 48201. E-mail: ssavasan@med.wayne.edu Received 10 August 2011; Accepted 23 January 2012 
HbF

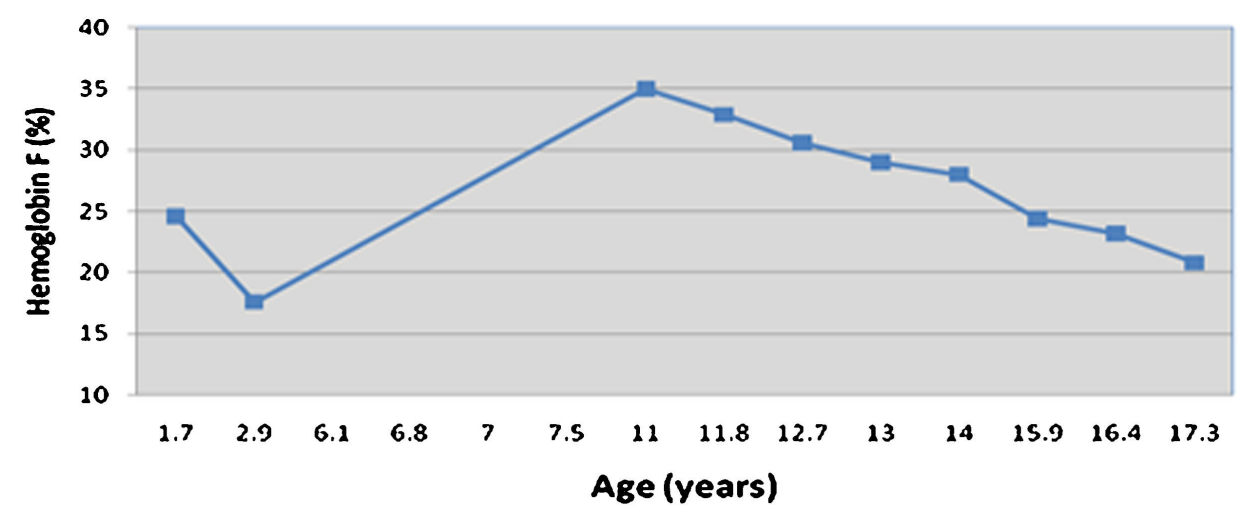

\section{MCV}

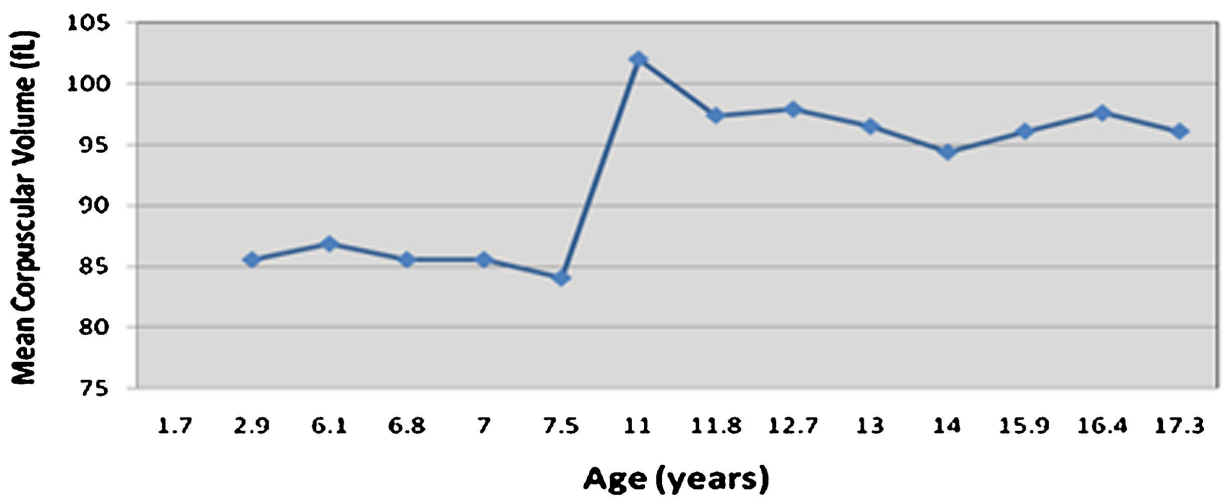

\section{MCH}

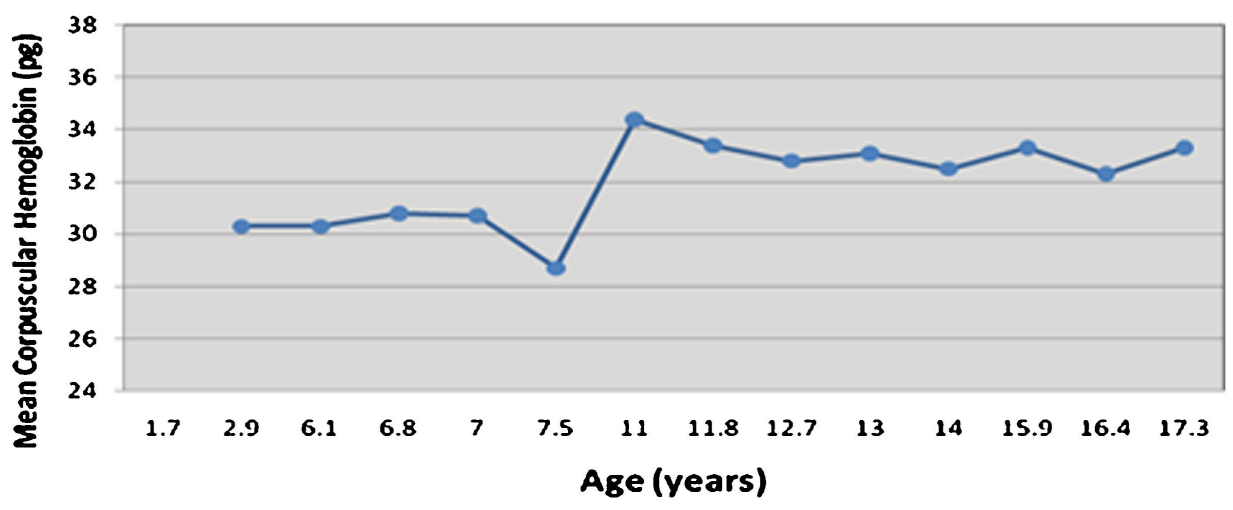

Fig. 1. Fetal hemoglobin (HbF), mean corpuscular volume (MCV), and mean corpuscular hemoglobin (MCH) changes over time. The patient's age in years is depicted on the x-axis. The values while the patient was undergoing chemotherapy were not included due to frequent packed red blood cell transfusions.

\section{REFERENCES}

1. Wilber A, Nienhuis AW, Persons DA. Transcriptional regulation of fetal to adult hemoglobin switching: New therapeutic opportunities. Blood 2011;117:3945-3953.

2. Long ZB, Oeffinger KC, Brooks SL, et al. Incidence and clinical relevance of abnormal complete blood counts in long-term survivors of childhood cancer. Cancer 2006;106:1634-1640.

3. Meier ER, Byrnes C, Weissman M, et al. Expression patterns of fetal hemoglobin in sickle cell erythrocytes are both patient- and treatment-specific during childhood. Pediatr Blood Cancer 2011;56:103-109.
4. Mabaera R, West RJ, Conine SJ, et al. A cell stress signaling model of fetal hemoglobin induction: What doesn't kill red blood cells may make them stronger. Exp Hematol 2008;36:1057-1072.

5. Sankaran VG, Menne TF, Scepanovic D, et al. MicroRNA-15a and -16-1 act via MYB to elevate fetal hemoglobin expression in human trisomy 13. Proc Natl Acad Sci USA 2011:108:15191524.

6. Bhanu NV, Trice TA, Lee YT, et al. A signaling mechanism for growth-related expression of fetal hemoglobin. Blood 2004;103:1929-1933.

7. Romero RR, Alberu J, Correa-Rotter R, et al. Serum erythropoietin levels in kidney donors after renal transplantation. Transplantation 2000;70:386-387. 